Lucrările Seminarului Geografic Dimitrie Cantemir

Vol. 44, October 2017, pp. 27-42

http://dx.doi.org/10.15551/Isgdc.v44i0.03

\title{
Un nouveau paradigme de l'innovation rurale: Apprendre des ruraux et des collectivités rurales
}

\section{Bruno Jean ${ }^{1}$}

1 Université du Québec à Rimouski, Canada

To cite this article: Jean, B. (2017). Un nouveau paradigme de l'innovation rurale: Apprendre des ruraux et des collectivités rurales. Lucrările Seminarului Geografic Dimitrie Cantemir, Vol. 44, pp. $27-42$. DOI: $10.15551 /$ lsgdc.v44i0.03

To link to this article: http://dx.doi.org/10.15551/lsgdc.v44i0.03 


\title{
UN NOUVEAU PARADIGME DE L'INNOVATION RURALE: APPRENDRE DES RURAUX ET DES COLLECTIVITÉS RURALES
}

\author{
Bruno Jean ${ }^{1}$
}

Résumé : Dans les représentations actuelles de l'innovation, les populations rurales ne sont pas considérées comme faisant partie de la classe créative. Cependant, l'histoire des communautés rurales du Québec montre que les ruraux ont été depuis longtemps et encore de nos jours, une classe créative, dont l'expérience se révèle riche d'enseignements. Les innovations rurales se manifestent dans les trois grandes dimensions du développement durable : aménager les environnements naturels, créer des instruments ou des institutions pour le développement économique et finalement, organiser la vie sociale. Ce nouveau paradigme de l'innovation rurale ${ }^{2}$ implique que nous puissions étudier les innovations rurales telles qu'elles émergent dans les communautés comme le fait l'Université rurale québécoise. Cela signifie aussi que l'on peut considérer ces communautés comme un patrimoine vivant, comme des «Livings Labs » comme en témoigne le programme des laboratoires ruraux du gouvernement du Québec qui s'intéresse déjà au développement de ces milieux avec la mise en place des Centres locaux de développement (CLD). Par ailleurs, on reconnaît qu'il existe des « rural clusters » qui constituent une autre forme d'innovation rurale.

\section{Introduction: l'innovation au cour des réalités rurales}

De nouvelles ruralités émergent et expriment un monde rural qui a toujours une place dans nos sociétés contemporaines en contribuant significativement à la prospérité collective. Les milieux ruraux sont des territoires d'avenir et non un vestige du passé survivant dans un monde post-moderne. Les communautés rurales sont en train de changer, de s'adapter, d'inventer de nouvelles formes d'une ruralité devenant plurielle. Pour comprendre ces ruralités d'aujourd'hui et de demain, nous devons projeter un nouveau regard sur cette réalité. Au Québec, ces nouvelles manières de regarder la ruralité émergent, en autres, avec les sessions de l'Université rurale québécoise qui est devenu un forum de l'innovation rurale en illustrant les nouvelles innovations sociales et institutionnelles mises en œuvre par les habitants des régions rurales.

\footnotetext{
${ }^{1}$ Professeur émérite - Université du Québec à Rimouski (UQAR), Canada

${ }^{2}$ La présent texte reprend certains éléments d'une présentation en anglais dans le cadre d'un colloque sur les politiques rurales de l'OCDE en Russie en 2012 et publié sous le titre : «A new paradigm of rural innovation: Learning from and with rural people and communities », dans OECD, Innovation and Modernising the Rural Economy, OECD Publishing, pp, 112-126, Paris, 2014. mCollection: OECD Rural Policy Reviews ISBN: 9789264205383 Version électronique: http://dx.doi.org/10.1787/9789264205390-en
} 
Le Québec rural engagé dans un processus de revitalisation, est devenu un véritable laboratoire vivant où les secteurs économiques traditionnels, comme l'agriculture et la foresterie, ont entrepris un processus de restructuration par la mise en marché de nouveaux produits et de nouvelles manières de produire.

Une nouvelle dynamique des interrelations rurales urbaines basée sur la complémentarité est en train de s'expérimenter. La renaissance rurale que l'on observe au Québec met en scène la culture comme un facteur de diversification économique. Les communautés rurales se mobilisent pour devenir les acteurs de leur propre développement et leurs efforts sont supportés par une politique appropriée.

Sans tomber dans la futurologie, on peut prédire sur la base de certains indices solides que des nouvelles ruralités se font jour. Cependant, les économies rurales sont mises au défi de répondre à la globalisation, aux nouvelles demandes urbaines, aux exigences gouvernementales, aux pressions environnementales et aux attentes des habitants ruraux euxmêmes. Tous ces défis impliquent un objectif commun de revitalisation des communautés rurales pour que celles-ci puissent contribuer au développement durable de toute la société.

Les ruraux sont aussi une «classe créative» capable d'aménager leurs environnements naturels, d'organiser leur développement économique et de planifier leur développement social. La ruralité devient alors un laboratoire qui participe à la construction de la société québécoise.

Les théories dominantes de développement économique postulent que seulement les grands ensembles urbains sont capables de générer l'innovation car ils attirent une classe créative soutenant leur croissance économique. Or, historiquement, les populations rurales ont été largement innovantes tant sur le plan environnemental que économique et social. Au lieu de penser qu'il faut apprendre aux populations rurales comment se développer, il faut plutôt se mettre à leur écoute et tirer les leçons de leur expérience. Les ruraux ont constamment mis en place des nouvelles organisations ou des nouveaux arrangements institutionnels pour faire face aux défis de chaque époque, par des adaptations économiques et des formes inédites de gouvernance locale capable de maintenir les services de proximités dont ont besoin de petites collectivités dispersées sur un vaste territoire.

L'histoire rurale du Québec illustre la créativité et l'inventivité des ruraux avec les rangs (routes rurales) comme mode de peuplement rural adapté à des territoires de faible densité, avec les corvées pour assurer la construction des granges et le maintien des chemins publics, avec les cercles agricoles assurant une forme originale d'auto-formation technique, avec les mutuelles incendies, avec les coopératives forestières sans oublier de mentionner d'autres innovations majeures comme les plans conjoints pour la mise en marché des produits agricoles - du commerce équitable avant la lettre - et les caisses populaires qui sont devenues une des grandes forces économique du Québec.

La ruralité contemporaine ressemble donc à un laboratoire vivant où s'inventent de nouveaux produits et où se mettent en place de nouvelles institutions pour faire face aux nombreux défis qui se posent aujourd'hui. Au Québec, les résidents de trois petits villages du Bas-Saint-Laurent, le JAL (Saint-Juste, Auclair et Le Jeune dans le Témiscouata) ont créé une nouvelle forme de coopérative, la coopérative de développement. Dans d'autres territoires forestiers, soumis à de nouvelles contraintes économiques, on a inventé la formule des Sociétés d'exploitation des ressources. Dans les zones agricoles, des coopératives d'utilisation en commun des machines agricoles ont vu le jour, et des Tables de concertation agroalimentaire régionale ont été mises en place. Pour maintenir les services éducatifs et 
sanitaires, des partenariats publics-privés et même publics-publics ont été créé. De plus, pour accompagner et supporter les dynamiques de développement local, des agences comme les Centres locaux de développement (CLD), les Sociétés d'aide au développement des collectivités (SADC) et les Fonds locaux de développement ont vu le jour.

L'innovation rurale ne concerne pas seulement l'innovation technique, bien visible dans les entreprises rurales, mais elle embrasse aussi l'innovation sociale. Plusieurs entreprises fabricant des machines agricoles ont prospéré en milieu rural avant de disparaître avec la mode des fusions. Cependant plusieurs manufacturiers de renommée sont nés en milieu rural avant de devenir de grandes corporations internationales: Bombardier, Tembec, Cascades, Prévost Car and Canam pour ne nommer que ceux-là. La revendication actuelle pour un accès internet haute vitesse partout sur le territoire rural du Québec manifeste la volonté de leurs habitants de tirer profit de ces nouvelles technologies d'information et de communication.

Les régions rurales sont fortement sensibles aux phénomènes économiques et sociaux contemporains comme la globalisation de l'économie et la généralisation de l'économie du savoir. Elles doivent restructurer et ajuster leur économie, probablement à un niveau plus grand que le reste de la société, ce qui met à mal la cohésion sociale typique des communautés locales. Les ruraux ne sont pas seulement des agriculteurs; ils occupent les campagnes et cette occupation du territoire remplit une fonction géopolitique essentielle car elle permet d'affirmer la souveraineté d'un État sur ce territoire. Comme les évolutions actuelles se traduisent par une dévitalisation et un questionnement sur l'occupation du territoire, une intervention politique pour contrer une telle situation devient justifiable.

Étant donné ce contexte, il n'est pas difficile de comprendre pourquoi la situation des régions rurales est devenue une préoccupation majeure pour le gouvernement du Québec qui y a répondu par la mise en place de la Politique nationale de la ruralité annoncée le 6 décembre 2001. Cette politique repose sur un modèle d'action qui postule la capacité des populations rurales à se mobiliser en vue d'un usage approprié des ressources disponibles dans leur milieu, en travaillant avec les MRC (Municipalité régionale de comté). Mais cette politique laisse sans réponse un certain nombre de questions : les dynamiques sociales et économiques qui sous-tendent le développement rural ont-elles été bien comprises et prises en compte? Comment les communautés rurales en forte dévitalisation pourront réellement aspirer à un développement durable?

\section{Comprendre que nous pouvons apprendre des communautés rurales}

Dans l'opinion publique comme dans les milieux scientifiques, une vision stéréotypée du monde rural conduit à penser que celui-ci se caractérise par un fort attachement aux valeurs traditionnelles et manque alors d'un sens de l'initiative, de la créativité et de l'innovation. Cette opinion tient lieu d'explication du retard du monde rural sur la route du développement. Or, nous devons changer ce regard sur la ruralité, biaisé par une sorte d'ethnocentrisme urbain. Les communautés rurales sont innovantes; de fait, l'innovation est pratiquement une nécessité vitale pour elles. Et si tel est le cas, leur expérience peut être utile pour les autres. Dès lors, toute la société peut apprendre des ruraux et des innovations rurales.

La fondation de l'Université rurale québécoise découle de cette prise de conscience, qui repose également sur une autre représentation de plus en plus reconnue, à savoir que le 
développement doit reposer sur les connaissances venant de l'expérience et des savoir-faire locaux. Nous pouvons apprendre des communautés rurales parce que les ruraux ont euxmêmes appris dans trois grands domaines d'action relevant d'un véritable développement durable: aménager avec prudence l'environnement naturel, assurer le développement économique et créer les conditions d'une vie sociale intéressante.

La créativité démontrée par les populations rurales se reflète dans un ensemble d'innovations sociales et institutionnelles qui ont été expérimentées avec succès. Elles sont nombreuses même si elles ne sont pas largement connues. Une des tâches principales de l'Université rurale québécoise est certainement de les faire mieux connaître pour le bénéfice de toute la société. Grâce à leur créativité, non seulement les populations rurales ont été capables de s'adapter au changement mais elles ont aussi offert une variété de modes de vie, de valeurs et de nouveaux modèles organisationnels et institutionnels adoptés par d'autres composantes de la société. Trop fréquemment, nous oublions que les Caisses populaires sont nées et se sont développées dans un environnement rural, et que les premières tentatives d'autogestion dans les entreprises ont eu lieu dans le monde rural en contribuant à définir ce modèle d'organisation du travail que l'on connaît de nos jours. Par ailleurs, nous observons souvent que les ruraux ont été capables de mettre en place des versions adaptées d'institutions économiques et sociales originellement pensées pour des milieux urbains.

Les conséquences du fait que la ruralité est une source d'expérience pour l'ensemble de la société sont nombreuses et profondes. Plutôt que de considérer les ruraux comme une partie du problème, nous devons plutôt les voir comme une partie de la solution. Le développement rural n'est pas un objectif à atteindre par l'application de mesures et de connaissances spécialisées venant de l'extérieur, mais plutôt un objectif que les populations rurales doivent assumer elles-mêmes. En d'autres termes, il est essentiel de comprendre que les populations rurales, comme d'autres populations, sont parfaitement capables de s'inscrire dans un processus d'apprentissage social qui assurera leur propre développement.

\section{L'innovation rurale: une caractéristique fondamentale de l'histoire rurale}

Les théories récentes sur le développement économique régional ont mis l'innovation (principalement technique) comme principal moteur de la création de richesse et des mesures de développement socio-économique par la croissance du PIB. Une telle interprétation de la croissance économique fait sens, mais la majorité de la recherche sur les théories associées de "systèmes d'innovation locaux ou régionaux" s'intéresse uniquement à l'innovation dans la période contemporaine et non dans le passé. Or, l'innovation a toujours été présente tant dans les zones urbaines que rurales mais son intensité a pu varier au cours des différentes périodes historiques.

Les innovations rurales dans le passé pourraient être caractérisées comme induites par la nécessité et non relevant de l'opportunité, selon la distinction classique proposée par le GEM (General Entrepreneurial Monitor). C'est ce que révèle l'histoire rurale du Québec marquée par de nombreuses innovations par nécessité. Tout d'abord, soulignons le mode de peuplement des terres par des propriétaires fonciers dans la société coloniale québécoise. La ferme a été dessinée comme très mince et long morceau de terre concédée à chaque famille. $\mathrm{Au}$ bout de chaque terre alignée sur un territoire donné, une route relie toutes les exploitations. Un seul chemin public peut alors desservir plusieurs exploitations agricoles configurées en bande étroite et qui se terminent à la route auprès de laquelle on installe la 
maison et autres bâtiments de la ferme. Ainsi, l'investissement public est efficace et les taxes pour chaque ferme jouissant de cette utilité publique est faible, même dans un pays reconnu comme grand et sous-peuplé.

Les mutuelles-incendie constituent une autre forme d'innovation rurale adoptée par nécessité. Il s'agir de coopératives assurant les risques d'incendies des bâtiments de ferme. En effet, s'agissant de constructions en bois, il était fréquent que des feux les détruisent complètement. Parallèlement avec une impressionnante solidarité qui faisait que tout le monde dans la communauté donnait du temps de travail et des ressources pour reconstruire ces bâtiments, tels que les granges, (ce que nous appelons «la corvée »), les habitants ont commencé à contribuer, avec de petites sommes d'argent, à un fond commun sur un base régulière auquel on recourt lors d'un incendie; Les ruraux québécois ont ainsi inventé ce qui est devenu une pratique usuelle de nos jours : la couverture par une assurance collective d'un risque. Dans les faits, ces mutuelles incendies ont d'abord été créées isolément dans chaque village, puis se sont fédérées pour devenir un élément d'une institution financière, les Caisses populaires. Ces dernières sont alors devenues une composante majeure du système bancaire québécois et de son économie.

Les coopératives du secteur agricole ont constitué une autre innovation très précoce du Québec rural; comme de nombreuses communes rurales sont isolées et à faible densité d'habitants, les fournisseurs privés d'intrants agricoles tout comme les entreprises de transformation des produits n'étaient pas intéressés à faire des affaires dans ces régions. Les agriculteurs n'avaient pas d'autres choix que de s'organiser eux-mêmes et se donner une organisation collective pour acheter leurs intrants et mettre leur production sur le marché. Au fil du temps, les nombreuses petites coopératives agricoles locales ont fusionné pour former un large conglomérat coopératif connu sous le nom de «La Fédérée » qui est devenue un joueur majeur de l'industrie agroalimentaire québécoise.

Durant les années d'après-guerre, les petits agriculteurs ont aussi innové en mettant en place des plans-conjoints qui organisent la vente collective (gestion de l'offre) de leurs produits; en réunissant les petites quantités produites par chaque ferme, ils se dotaient d'un pouvoir de négociation pour commercialiser leurs produits à des acheteurs de plus en plus oligopolistiques. Les petits producteurs agricoles se sont ainsi donnés une capacité de négociation face aux grandes corporations agro-industrielles. Cette innovation, proche du commerce équitable, a permis de maintenir les fermes familiales qui recevaient ainsi un meilleur retour sur leur production. Dans le cas de la production laitière, fort répandue au Québec, la part qui retourne au producteur agricole atteint jusqu'à 40-50\% du prix payé par le consommateur final au lieu de seulement $10 \%$ comme c'est généralement le cas en agriculture toutes productions confondues. Mais cette « gestion de l'offre » a débouché sur un conflit inattendu au sein du monde agricole, au moment où les grandes coopératives laitières devenaient des acteurs majeurs de la transformation et de la commercialisation du lait. A travers cette innovation, les producteurs agricoles deviennent, simultanément des vendeurs (comme partenaires d'un plan-conjoint de mise en marché) et des acheteurs (comme membres de la coopérative) de leur propre production. Cette situation conflictuelle a été résolu d'une manière intéressante : Afin de maintenir un prix élevé pour le lait demandé par les producteurs, les coopératives sont rapidement devenues très technologiquement performantes ; cela leur a permis de réduire leurs coûts de production, et de restituer les gains de productivité en découlant, tant aux agriculteurs qu'aux consommateurs de produits laitiers. 
La ruralité québécoise se caractérise par la forêt et un tiers des communautés rurales en dépendent pour leur prospérité économique. Dans les régions habitées, la forêt est souvent privatisée et elle est aux mains d'une multitude de petits propriétaires qui la cultivent. Dans d'autres régions largement inhabitées, la forêt reste une propriété publique (crown lands), son exploitation étant confiée, contre redevances, à des grandes corporations forestières capables d'acheter les droits de coupe au Gouvernement du Québec. Autrefois, ces grandes sociétés, souvent étrangères, recherchaient des petits entrepreneurs locaux pour assurer la récolte du bois. Toutefois, dans plusieurs collectivités, on a mis sur pieds des «chantiers coopératifs », une coopérative de travailleurs exécutant ce travail en forêt pour le compte de ces grandes corporations. Organisés en coopératives, ces travailleurs étaient capables de négocier de meilleures conditions pour le même travail qu'ils avaient l'habitude de faire avec les petits entrepreneurs locaux. De nos jours, une fédération de ces coopératives forestières est toujours active.

À la fin du $19^{\mathrm{e}}$ siècle, l'agriculture québécoise est devenue davantage commerciale avec la production et la vente de beurre et de fromage (le fameux cheddar) pour le marché britannique. Il est alors devenu clair que les fermiers devaient améliorer leurs méthodes de production spécialement pour rehausser la qualité du lait livré aux nombreuses petites fromageries présentes dans tous les villages. Ce besoin a suscité la création des «Cercles Agricoles » dans chaque collectivité pour dispenser une formation de base en production laitière, selon un modèle d'auto-formation qu'on appelait alors « l'enseignement agricole mutuel ». Comme la profession d'agronome n'existait pas encore à cette époque, les agriculteurs ont décidé d'apprendre d'eux-mêmes, en partageant leurs expériences dans ces clubs agricoles d'auto-formation.

\section{L’innovation rurale contemporaine: sous-estimée, diversifiée et contribuant à un développement rural durable}

Après avoir dressé la liste des innovations rurales les plus significatives au Québec, il apparaît possible de les classifier selon les trois grandes dimensions du développement durable : 1) aménager de manière productive et prudente les ressources naturelles : 2) assurer le développement économique; 3) organiser une vie humaine acceptable socialement. La plupart de ces innovations sont des innovations sociales car elles passent par des créations et des expérimentations de nouveaux arrangements socio-institutionnels dans le but de résoudre un problème, d'organiser la prestation d'un service ou de profiter d'une nouvelle opportunité de création de richesse pour le mieux-être collectif.

Pour chacune de ces dimensions, nous proposons dans les pages suivantes un inventaire des innovations spécifiques ayant abouti à la création d'institutions nouvelles en milieu rural.

\section{1) Assurer un usage productif et durable des ressources naturelles}

- L'Organisme de gestion en commun (OGC) de la forêt privée et le Résam Regroupement des sociétés d'aménagement forestier du Québec : Ces deux nouvelles institutions visent à assurer une meilleure gestion et une meilleure productivité des forêts privées. Ces organismes peuvent aussi réhabiliter la forêt publique à proximité des communautés afin de créer des emplois dans les collectivités rurales qui dépendent de la forêt. 
- La Réserve Duchénier (réserve faunique) : Cette nouvelle institution a pour mission d'assurer la gestion d'une grande partie du domaine public pour la récréation, les loisirs, le tourisme et la conservation de la faune tout en créant des opportunités d'emplois. Il s'agit du seul exemple au Québec où une « réserve faunique » est gérée par les citoyens.

- Les Clubs conseils agro-environnementaux en agriculture : Il s'agit d'un nouvel arrangement institutionnel où un groupe d'une cinquantaine d'agriculteurs se réunissent ensemble dans une association qui embauche un agronome dans le but de développer les meilleures pratiques en ce qui concerne la conservation de sols et des ressources naturelles.

- OBV : les organismes de bassin-versants (gestion de l'eau) : Cette nouvelle institution permet aux différents partenaires présents dans un bassin-versant donné de s'associer pour mettre en place une gestion durable des eaux du bassin versant en question.

\section{2)Assurer leur développement économique}

- Le crédit populaire avec les Caisses populaires: nous avons vu plus haut que les Caisses populaires ont été présentes dans toute l'histoire rurale du Québec. Ces banques coopératives caractérisent aussi la période actuelle en soutenant le développement économique du secteur rural.

- Les Coopératives de développement (le JAL) : Il s'agit d'une nouvelle forme de coopérative dont la mission est d'améliorer la situation économique et sociale locale dès lors que tous les citoyens de trois communautés rurales en deviennent membres.

- Les coopératives d'utilisation du matériel agricole en commun (CUMA) : Cette nouvelle forme de coopérative permet à de petits producteurs agricoles de se mettre ensemble pour acheter et utiliser diverses machines agricoles ne servant que quelques jours par année. Il en est attendu une réduction des coûts de la machinerie, afin d' assurer la survie des petites entreprises.

- Les SER (Sociétés d'exploitation des ressources): Cette nouvelle institution a été mise en place dans le contexte des luttes sociales rurales qui ont caractérisé les années soixante-dix. Leur rôle est de permettre aux populations rurales vivant dans les communautés à proximité de la grande forêt publique québécoise d'avoir un accès aux ressources forestières publiques en y réalisant des travaux de sylviculture et d'entretien.

- Le Réseau des Sociétés d'aide au développement des collectivités (SADC) : Ce réseau d'agences de développement local a été créé par le gouvernement fédéral (connu sous le nom de CFDC: Community Futures Development Corporation) selon un mécanisme de dévolution de sa gestion à un regroupement de partenaires locaux, en vue d'offrir divers programmes d'assistance technique et financière pour le démarrage des petites entreprises. Ils agissent comme une banque communautaire qui s'avère très performante au regard du taux élevé de survie des entreprises supportées. 
- Les Conseils locaux de développement (CLD): Ils permettent aux gouvernements locaux des Municipalités régionales de Comté d'offrir des aides financières et techniques pour soutenir le développement économique local. Nous décrirons davantage cette institution novatrice plus loin.

- Les Fonds locaux de développement (les Solides): en lien avec les CLD et les municipalités, ces fonds locaux de développement aident au financement $\mathrm{du}$ démarrage des petites entreprises.

- Le Rural cluster: Il se définiti comme un système d'innovation locale dans une petite région rurale donnée comme le cas de La Pocatière qui sera décrit plus loin.

3) Organiser une vie humaine acceptable socialement.

- Les Coopératives de solidarité et de santé : Elles sont habituellement mises en place dans un centre de santé ou un centre communautaire local pour attirer les médecins en offrant la gestion de la clinique médicale; ces coopératives offrent un accès à un médecin non seulement à leurs membres mais à tous les citoyens.

- Les partenariats publics-publics pour le maintien des services de proximité : Ce nouvel arrangement institutionnel où au moins deux administrations publiques, comme la Commission scolaire et le Conseil municipal, s'associent pour leur bénéfice mutuel a été institué pour réduire les coûts d'opération des édifices publics qu'ils gèrent en optimisant l'usage des espaces. Il est fréquent de voir des partenariats publics-privés pour assurer la présence de certains services publics dans les communautés rurales (comme la poste) mais ce qui nouveau et innovant ici, ce sont ces partenariats publics-publics (PPP) au lieu des traditionnels PPP comme partenariat public-privé.

- L'accès Internet comme service public : Il s'agit d'un accès Internet haute vitesse offert, à titre de service public, par le gouvernement local à tous les citoyens à un prix très bas. Cela est rendu possible même dans des zones rurales éloignées par la technologie Wi-max comme on peut le voir dans la municipalité de Nouvel en Gaspésie.

- Les Tables de concertations agroalimentaires: un nouvel arrangement institutionnel où les différents partenaires agroalimentaires d'une région donnée se réunissent pour améliorer le secteur agroalimentaire en supportant de nouvelles productions, de nouveaux procédés de transformation pour augmenter la valeur ajustée et soutenir aussi la mise en marché de ces produits.

- Les Écoles primaires multi-niveaux : Ce nouvel arrangement institutionnel est une réponse à la baisse du nombre d'enfants dans une école primaire locale donnée. Souvent, on regroupe les élèves des deux cycles des six ans d'étude du primaire en deux classes, ce qui permet de maintenir l'école ouverte.

- Les Ententes inter-municipales de service: un nouvel arrangement institutionnel par lequel des gouvernements municipaux (souvent de très petites collectivités rurales) s'unissent pour mutualiser l'offre d'un service donné (comme la protection-incendie ou la gestion des matières résiduelles) 
Plusieurs des innovations mentionnées ici sont largement répandues dans les collectivités rurales comme les Caisses populaires ou les Ententes intermunicipales de service. D'autres innovations sont relativement uniques comme la Réserve Duchénier car elles sont difficiles transmissibles ou reproductibles en milieu rural. Notre liste n'est pas exhaustive car il s'agissait simplement de montrer par plusieurs exemples que l'innovation rurale est bien une réalité observable et riche d'enseignements. Afin d'expliciter ceux-ci, nous nous proposons de faire un focus sur un cas particulièrement significatif : celui des CLD.

\section{Une organisation vouée au développement local: les Centres Locaux de Développement (CLD)}

Comme le gouvernement fédéral dans les années 1990, le gouvernement du Québec a adopté une approche de développement axée sur le local comme cadre d'intervention de ses politiques publiques. Cela impliquait la mise en place d'un nouvel organisme voué au développement local, le Centre local de développement (CLD) dans chaque Municipalité régionale de Comté (MRC). Les CLD ont d'abord été établis comme des organisations à but non lucratif gérées par des représentants socio-économiques locaux, mais dans une récente réforme, ils sont devenus une partie intégrante des MRC. Ils offrent un soutien aux entrepreneurs locaux et ils sont chargés de rédiger et d'adopter un plan d'action locale pour le développement économique et la création d'emplois (Plan d'action locale pour l'économie et l'emploi, ou PALÉE). La compétence d'un CLD semble faire double emploi avec le mandat et le territoire d'intervention des Sociétés d'aide au développement des collectivités (SADC) mis en place dans les années 1980. La complexité de ces organisations et des arrangements institutionnels, avec leurs inévitables degrés de chevauchement, de duplication, de concurrence et de tension n'est pas atypique dans un contexte de développement rural. Fuchs et Shapira (1995) ont noté cette tendance dans le développement rural dès 1995. L'Irlande vient de traverser un processus de restructuration radicale pour réduire le nombre d'agences de développement rural de plus de 100 à 55. L'Union européenne continue de lutter contre la prolifération des agences de développement. La réorganisation institutionnelle des CLD, en les mettant sous l'autorité des municipalités régionales de comté (MRC), a généré bien d'autres conséquences, qui sont examinées cidessous. Les MRC doivent confier l'exercice de leurs nouvelles responsabilités en matière de développement local à un organisme sans but lucratif constituée en vertu de la partie III de la Loi sur les compagnies du Québec.

Dans les zones rurales, les affaires commerciales du CLD sont gérées par un conseil d'administration composé d'élus municipaux des municipalités locales, déjà membres du Conseil de la MRC, et de représentants de l'entreprise et de l'économie sociale. Tous les membres du conseil d'administration du CLD sont nommés par le conseil des maires de la MRC. Le député, le directeur général du CLD et le directeur du Centre local d'emploi présent sur le territoire de la MRC sont également membres du conseil d'administration, mais ils n'ont pas droit de vote. Le gouvernement du Québec et chacune des MRC contribuent conjointement au financement des activités de la CLD, qui doit présenter un rapport d'activités et les états financiers, conformément aux conditions énoncées par les MRC.

Les CLD offrent une assistance de première ligne et des services d'assistance technique ou financière auprès des entrepreneurs potentiels ou actifs, ainsi que à destination 
des individus ou des groupes, ou encore des entreprises d'économie sociale. Ces services comprennent la consultation, l'orientation, l'aide à la préparation des plans d'affaires, y compris les études de préfaisabilité. Mais aussi, ils aident au financement et à la gestion financière des projets des entreprises, principalement par le biais Fonds local d'investissement (fonds d'investissement de proximité), à la formation à l'entrepreneuriat, au mentorat etau suivi des entrepreneurs et des entreprises, et à l'orientation vers des services plus spécialisés, comme les services de développement technologique ou d'exportation. Le mandat des CLD se chevauche avec celui de la SADC. Dans certaines MRC, l'ajustement a été assez difficile, même si, dans la plupart des cas, les CLD et les SADC ont appris à bien travailler ensemble. Parfois, les deux organisations sont situées dans le même bâtiment, offrant aux entrepreneurs locaux un "guichet unique".

Les CLD sont chargés de concevoir et mettre en œuvre diverses mesures d'aide financière pour soutenir le développement des entreprises et des projets de développement local. Ces mesures font partie du Plan local pour l'économie et l'emploi du CLD et elles doivent stimuler l'économie, créer des emplois et de développer l'esprit d'entreprise. Les mesures visent essentiellement les jeunes entrepreneurs, le développement des entreprises d'économie sociale et la diversification économique. Le Fonds local d'investissement (fonds d'investissement local, ou FLI) est le principal instrument financier de la CLD. Il offre du financement (prêts, une aide financière) aux entrepreneurs pour le démarrage et l'expansion d'entreprises, y compris les entreprises d'économie sociale. La planification dans un CLD est principalement axée sur le développement économique local, tandis que le mandat de la MRC est l'aménagement du territoire. Néanmoins, il est clair que le débat autour de la construction du plan agit souvent comme un processus d'apprentissage dans lequel les différentes parties prenantes acquièrent une meilleure compréhension de leur région, de ses atouts et des défis, ainsi que d'une meilleure représentation de ce qui pourrait être entrepris, au niveau local, pour favoriser un développement rural plus durable. Il est également prévu que ce processus mènera à des actions de développement plus stratégiques et intégrées.

\section{Un cluster rural le cas de La Pocatière}

Les deux sections suivantes présentent deux innovations rurales, l'une concernant la création locale d'emplois et le développement des entreprises sur un territoire, et la seconde sur la formation des acteurs de développement du monde rural. Les deux peuvent être compris non seulement comme des innovations techniques, éducatives ou entrepreneuriales, mais aussi des innovations sociales.

Selon une hypothèse scientifique répandue, un système régional d'innovation doit rayonner à partir d'un grand centre urbain. Néanmoins, la recherche d'un éminent spécialiste canadien dans le domaine, David Doloreux, démontre que les résidents ruraux peuvent établir un système local ou régional d'innovation. L'exemple de La Pocatière, une ville d'environ 5000 habitants entourée de petites communautés agricoles et rurales, illustre la trajectoire de développement d'un système local d'innovation dans une région rurale dans une perspective historique longue (1830-2005) et le contexte institutionnel qui il est apparu (Doloreux, Dionne et Jean, 2007).

Les attributs d'un système local d'innovation identifiés dans la littérature récente sont présents à La Pocatière. Ils soulignent la pertinence des acteurs institutionnels et de leur capacité à répondre aux mutations économiques et technologiques. Les acteurs 
institutionnels de La Pocatière, comme le montre une analyse dans la longue durée historique, se sont adaptés aux changements continuels et radicaux dans la configuration institutionnelle, et en particulier dans la période contemporaine, à la multiplication des structures de toutes sortes, souvent publiques ou quasi-publiques. La prédominance des acteurs du secteur public dans cette configuration institutionnelle a été observée dans d'autres systèmes locaux d'innovation (Cooke et al., 2004). Ce qui est remarquable dans ce cas, c'est l'importance d'une action concertée entre les acteurs publics et privés, fondée sur un patrimoine de structures de soutien local et des aides publiques qui sous-tendent le système local d'innovation.

Le système local d'innovation de La Pocatière suit une trajectoire de développement originale et remarquable. Contrairement à de nombreux systèmes locaux d'innovation dans les régions de tradition industrielle ancienne, La Pocatière n'a pas de passé industriel. Ce système local d'innovation a émergé d'une petite ville rurale ayant une solide tradition de recherche agricole et de transfert des connaissances avec l'enseignement public, la recherche appliquée et de transfert technologique. Ce lien impliquait un système de production composé non pas par des entreprises, mais plutôt par une multiplicité de producteurs isolés. La classe agricole a non seulement constamment assimilé et intégré toutes les innovations liées au développement de l'agriculture depuis le milieu du XIXe siècle, mais elle a vécu la transition de l'agriculture de subsistance et à l'agriculture familiale et à une agriculture commerciale et industrielle intégrée dans le système agroalimentaire mondial. C'est seulement par la suite, et même assez récemment, que ce système local d'innovation s'est diversifié dans la production industrielle, avec l'avènement de l'usine de Bombardier à La Pocatière, les activités de recherche sur les technologies physiques appliquées du Cégep (Collège d'enseignement général et professionnel), et l'émergence de plusieurs entreprises spécialisées comme sous-traitants. L'analyse de ce cas montre comment un système local d'innovation peut dépendre de la coopération inter-institutionnelle plutôt que de la coordination entre les entreprises.

Le fonctionnement et la dynamique du système d'innovation de La Pocatière illustrent l'importance des réseaux locaux, qui étaient en mesure de mobiliser des ressources extérieures, à la fois politiques et économiques, à des échelles variées (régionale, provinciale, fédérale et internationale) dans la poursuite de leurs activités d'innovation, et de renforcer et positionner stratégiquement leur cadre institutionnel.

Les zones rurales et périphériques sont souvent considérées comme peu exposées à l'introduction et au développement des systèmes d'innovation. La Pocatière, à partir du milieu du XIXe siècle, était incontestablement un centre important du développement technologique, fortement intégrée avec le centre du Québec et relié à d'autres centres internationaux importants, notamment en Europe. Dans la seconde moitié du XXe siècle, le déclin relatif de La Pocatière sur le plan technologique n'a pas réduit sa capacité à soutenir son développement en tant que système d'innovation et cette ville n'est pas tombée dans l'isolement technologique, institutionnel et social. Sur une longue période, de nombreuses institutions publiques locales de La Pocatière, exceptionnellement, ont été en mesure de déployer des stratégies communes visant à un projet de développement économique à l'échelle du territoire.

Cependant, à travers tous les ralentissements ou les crises économiques, les différents agents et les organisations ont pu se transformer à long terme et influencer le fonctionnement et la capacité d'évolution du système d'innovation. Par exemple, le 
déménagement de la Faculté d'agriculture de l'Université Laval de la ville de Québec n'a pas mis fin aux activités de recherche de la Ferme expérimentale fédérale. Au lieu de cela, un technopôle agro-alimentaire et agro-environnemental régional centré sur La Pocatière a été développé, et un réseau de petits centres de l'agroalimentaire misant sur des savoir-faire et le transfert de technologie a prospéré pendant environ une décennie autour du Centre de développement Bioalimentaire du Québec (CDBQ). L'arrivée de l'usine Bombardier et la création d'une institution publique comme le CÉGEP et de ses Centres de transfert de technologie, qui ne faisaient pas initialement partie des plans du gouvernement, mais qui ont été obtenues grâce à la mobilisation des élites locales, sont d'autres exemples. Ils illustrent clairement une nouvelle expansion de ce cluster rural, cette fois avec l'aide d'un acteur majeur du secteur manufacturier privé et les organismes de liaison technologiques coordonnés avec l'industrie.

\section{L'Université rurale québécoise: mettre en valeur les innovations rurales}

L'Université rurale québécoise (URQ) est une organisation informelle que j'ai aidée à mettre en place en 1997 avec d'autres professeurs de l'Université du Québec et des membres des trois réseaux d'acteurs dédiés au développement rural, y compris Solidarité rurale du Québec, le Réseau des SADC (Sociétés d'aide au Développement des Collectivités Community Futures au Canada) et les Centres locaux de développement (CLD). Tous les deux ans, l'Université rurale organise un forum d'échange et de formation pour les acteurs du développement rural. Sa mission est de soutenir le développement des régions et des communautés rurales en initiant des activités de formation continue pour les acteurs et les agents de développement rural, basée sur une approche «partage des connaissances» visant à générer des actions réfléchies et des réflexions agissantes. Au fil du temps, même si cela n'est pas explicitement exprimé dans son mandat, l'Université rurale, qui est déjà en soi une innovation dans sa configuration institutionnelle unique, a été en mesure de mettre en valeur l'innovation rurale.

Le modèle URQ est basée sur une « fertilisation croisée des connaissances », définie comme une approche pédagogique informelle, conviviale construite sur l'hypothèse qu'il est possible d'apprendre de la pratique, de l'action grâce au savoir expérientiel ayant autant de valeur que le «savoir savant». Son objectif est de permettre un échange entre la connaissance «académique» (émergeant de réflexion) et de la connaissance «fondée sur l'expérience» (émergeant de l'action) (Jean, 2004). Sa perspective est décidément à long terme, car le développement des capacités communautaires, son objectif déclaré, ne peut pas être mesurée sur le court terme. Lavergne et Saxby (2001, p.3) soulignent que le principal défi en matière de développement des capacités consiste à concilier le besoin immédiat de résultats tangibles (par exemple à des fins de reddition de comptes) et les besoins à long terme en matière de renforcement des capacités dans un contexte de développement durable.

L'Université rurale, c'est un événement unique et bien plus qu'un symposium où les participants viennent chercher des informations. Les données recueillies lors des évaluations montrent que sa formule offrant des activités mixtes (conférences, ateliers, excursions, etc.) et les échanges qu'elle permet entre partenaires issus de divers milieux sont considérés comme très enrichissants. L'Université rurale est devenue un événement majeur dans la vie rurale québécoise. En ce sens, l'Université rurale, semble répondre à un véritable besoin. Elle 
contribue également à se rapprocher de son objectif: le développement des capacités communautaires des collectivités rurales

Le caractère apolitique de l'Université rurale constitue une autre caractéristique intéressante. Le développement communautaire est souvent influencé par les luttes politiques qui émergent dans les organisations de développement, en isolant les acteurs plutôt que de les rassembler. Beaucoup de gens ont noté le «terrain neutre» que l'URQ, notamment en termes d'échanges riches et de partage d'informations concrètes.

Enfin, la flexibilité du modèle est un de ses atouts. Mis à part un Comité de pilotage composé de professeurs de l'Université du Québec et des représentants des réseaux de développement actifs en milieu rural, l'Université rurale n'a pas de structure organisationnelle permanente. À ce jour, chaque session de l'Université rurale a été organisé en mobilisant les structures existantes (organisations de développement, universités, etc.) Avec moins de pesanteurs bureaucratiques, ce mode d'organisation rend plus facile l'amélioration du modèle ou, le cas échéant, la remise en question de sa pertinence - même. L'opportunité de la tenue d'une URQ peut donc être réévaluée avant chaque événement. Cette approche organisationnelle organique n'est pas sans ses défis. Par exemple, il est difficile de mettre en commun et de coordonner l'information, en particulier des informations utiles pour les évaluations. La collecte de fonds est aussi lourde, parce que les demandes doivent être réitérées par différentes personnes et organisations.

Ces deux cas (Université rurale et les CLD) montrent que la population rurale a la capacité de faire face aux défis auxquels elle est confrontée. Le système local de production de La Pocatière montre que les dirigeants de cette ville rurale peuvent mettre en place des systèmes d'innovation locaux ou régionaux dans un cadre rural. Cet exemple peut être utile pour le développement local dans de nombreuses autres régions. L'Université rurale montre comment la population rurale peut se mobiliser pour une initiative qui leur donne accès à l'éducation et à la formation dans le développement rural en partageant durablement leurs connaissances. Ces deux expériences valident l'idée que le développement rural peut se produire, comme une résultante du renforcement des capacités des populations rurales.

\section{Les ruraux: une classe créative ou innovante?}

Les théories récentes du développement économique mettent l'emphase sur les effets de la proximité, ce qui évident dans les zones urbaines, ou encore métropolitaines. Richard Florida a expliqué ce processus par le rôle d'une supposée classe créative. La créativité serait liée à une population ayant un haut niveau de formation ou de scolarité et un esprit ouvert au changement, ce qui serait le cas dans les zones urbaines multiculturelles, Mais alors peut-on poser la question : les ruraux seraient-ils moins créatifs?

Plusieurs faits démontrent la créativité et l'inventivité des populations rurales, en dépit de la thèse qui veut que l'éducation soit un prérequis pour l'innovation. Nous devons alors changer de paradigme et se demander ce que nous pouvons apprendre des ruraux en observant leur capacité à innover, à stimuler le développement économique, et à améliorer la qualité de vie dans les campagnes. Cependant, l'innovation rurale diffère des modèles d'innovation observables dans les environnements urbains. L'économie des régions rurales est basée sur les ressources naturelles. Les innovations rurales en agriculture s'expriment à travers de nouvelles formes d'agriculture produisant de nouveaux produits dits « de qualité » qui reconnectent les habitants des villes et les agriculteurs. Cette nouvelle agriculture qui met 
l'emphase sur des produits biologiques ou de spécialité prend de plus de place dans l'espace rural. Dans certaines régions délaissées par l'agriculture traditionnelle, ou productiviste, cette nouvelle agriculture «du terroir » est en train de faire revivre l'économie de ces territoires.

Des opportunités émergent avec le changement des habitudes de consommations comme la tendance à « manger local ». La mise en place de marchés agricoles publics locaux a stimulé les capacités innovatrices d'une nouvelle génération de producteurs agricoles qui sont souvent eux-mêmes issus des milieux urbains et qui ont fait le choix de la ruralité. Ce renouvellement d'intérêt pour la production agricole explique par exemple l'essor d'une offre de fromages fins, ou de spécialité, qui entre en compétition avec des fromages européens importés. Le développement d'une agriculture en harmonie avec la terre et l'intérêt des citoyens pour les productions locales permettent de revaloriser les potentialités de l'agro-écologie et de renouveler les termes du contrat social entre les producteurs agricoles et le reste de la société.

Des nombreuses innovations ont eu lieu dans 1'exploitation de la forêt, et émergent de nouveaux modèles innovants de gestion des forêts publiques par les communautés rurales. Le défi actuel du secteur forestier québécois est de reconnaître le potentiel d'une approche multi-usage de notre forêt comme une source diversifiée de produits et de mieux comprendre son rôle dans l'environnement, les loisirs et le tourisme. Des exemples de tels potentiels inexploités concernent les applications du bois pour les revêtements de sol à l'aide de résidus de bois jusqu'alors mis au rebut et l'utilisation de revêtements en bois pour habiller l'extérieur de la maison.

La diversification de l'économie de la forêt comprend également des produits forestiers non ligneux (PFNL). Ce sont des produits autres que le bois qui proviennent de sources biologiques de la forêt et nécessitent peu de transformation. Ils peuvent permettre aux collectivités forestières de bénéficier des ressources naturelles situées à leur porte. Cette catégorie comprend également l'acériculture, la production d'arbres de Noël, les bleuets sauvages (myrtilles), les champignons et les huiles essentielles extraites de conifères. Plus de 400 produits potentiels pourraient être récoltés à partir de nos forêts et être introduits dans un marché de plus en plus sensible à l'intérêt nouveau des consommateurs pour les produits biopharmaceutiques ou de neutraceptiques (compléments alimentaires naturels). Avec les PFNL, le potentiel de la biomasse forestière à être utilisé pour l'énergie et la production de biocarburants est également devenu une réalité. Les PFNL peuvent ainsi réduire notre dépendance au pétrole et de diminuer les émissions de gaz à effet de serre (GES), tout en élargissant la gamme des avantages socio-économiques que les forêts offrent à la société.

Enfin, les emplois industriels peuvent être exportés vers la campagne. Le gérant d'une grande brasserie à l'extérieur de Vancouver a révélé que la main-d'œuvre rurale pourrait être un atout qui peut compenser les coûts de la distance et du transport. Non seulement les travailleurs ruraux ont tendance à être moins absents au travail, mais quand les machines tombent en panne, ils peuvent trouver des solutions immédiates ou faire des réparations temporaires en attendant l'arrivée de nouvelles pièces de rechange. En conséquence, la ligne de production ne s'arrête pas aussi souvent qu'en ville, où les travailleurs auraient attendu les pièces et des techniciens pour exécuter la réparation, d'où une baisse de productivité. Une telle ingéniosité montre comment la culture rurale se distingue, en dépit de sa prétendue fusion avec la culture urbaine. 
Pour évaluer l'innovation rurale, il faut prendre en considération que la population rurale ne représente que $20 \%$ à $25 \%$ de la population totale. Il n'est donc pas surprenant que le secteur rural génère moins d'innovation. Souvent, les innovations techniques sont développées par des firmes d'ingénieries basées en ville pour une utilisation en milieu rural. Un exemple est la traditionnelle mousse de tourbe, qui peut servir de bio-filtration pour les systèmes d'eaux usées dans des maisons isolées. Tout cela montre comment les usages des ressources naturelles en milieu rural peuvent être ré-imaginés et réutilisés. Une vaste opération de recherche-développement est nécessaire pour trouver de nouvelles utilisations pour ces ressources. Cela montre bien que l'innovation sera au cœur de l'évolution future de l'économie rurale.

\section{Conclusion}

Selon la distinction classique proposée par le General Entrepreneurship Monitor, certaines personnes deviennent entrepreneurs par nécessité, d'autres par opportunité. Si on applique cette distinction entre les innovations dans le secteur rural, il semblerait que les innovations passées se sont généralement produites en réponse à la nécessité. Par exemple, les individus ont créé des coopératives, car les entreprises privées n'étaient pas prêtes à offrir les produits ou les services dont ils avaient besoin. Cependant, de nos jours, l'innovation dans le milieu rural correspond plus à l'innovation par opportunité. Il n'y a pas assez d'espace ici pour discuter des modèles de propriété liés à ces innovations rurales, mais elles sont diverses, allant des entreprises privées classiques aux coopératives et aux entreprises collectives typiques de l'économie sociale. De nombreuses entreprises de ce genre démarrent des activités de type culturel. Le développement de la culture en milieu rural avec des écomusées, les centres d'interprétation et les théâtres d'été est également devenu un secteur économique dynamique dans certaines régions rurales.

La ruralité peut aussi donner prise à des «Laboratoires ruraux » au sens d'un modèle d'action pour soutenir l'innovation, comme une méthode pour susciter des innovations dans plusieurs domaines. Durant la seconde phase de la Politique nationale de la ruralité du Québec, politique rurale reconnue par l'OCDE, cette formule de «Laboratoire rural » a été reconnue, à travers un appel à projets en vue de l'octroi de subventions substantielles à des propositions prévoyant des retombées économiques et sociales significatives. Plus d'une trentaine de laboratoires ruraux sont en cours d'expérimentation. Même si plusieurs initiatives ne vont pas passer le test de la réalité, ce programme des Laboratoires ruraux peut être considéré comme un succès parce que les innovations qui vont survivre et se développer vont contribuer grandement à la qualité de vie et la prospérité des communautés rurales où ces expérimentations auront été menées. Plusieurs innovations réussies pourront aussi être transférables à d'autres collectivités rurales.

Toutes les innovations rurales décrites plus haut confirment que de nouvelles ruralités émergent. Le monde rural prend sa place dans les sociétés contemporaines et peut contribuer à la prospérité collective. Les régions rurales sont des territoires d'avenir, et non les survivances ou les vestiges du passé dans un Québec moderne. Les collectivités rurales changent, s'adaptent, innovent, inventent de nouvelles formes de ruralité fort variées. Au Québec, de nouvelles manières de voir la ruralité se font jour, notamment à travers les sessions de l'Université rurale québécoise qui devenue un forum de l'innovation rurale. 
La ruralité québécoise s'engage dans un processus de revitalisation. Elle est devenue un laboratoire grandeur nature où les secteurs économiques traditionnels comme l'agriculture et la foresterie se restructurent en inventant de nouveaux produits et de nouvelles manières de faire en utilisant des technologies de pointe. De nouvelles relations, basées sur une meilleure compréhension de la complémentarité rurale-urbaine et de l'occupation du territoire, apparaissent. La renaissance rurale au Québec repose aussi sur la culture comme un facteur de développement et de diversification économique. Les communautés rurales se mobilisent pour devenir les acteurs de leur propre développement et leurs efforts sont supportés par une politique rurale appropriée.

\section{Références bibliographiques}

1. Conference Board of Canada ,2009. Les communautés rurales. L'autre moteur économique du Québec, Ottawa, Conference Board of Canada, 139 pp.

2. Cooke P., Heidenreich M. and Braczyk H.-J.,2004. Regional Innovation Systems: The Role of Governances in a Globalized World, second edition, Routledge, London.

3. Doloreux D. and Dionne S.,2007. Évolution d'un système local d'innovation en région rurale: Le cas de La Pocatière dans une perspective historique, 1827-2005. UQAR - Rimouski, Éditions du CRDT

4. Doloreux D., Dionne S. and Jean B.,2007. The Evolution of a local Innovation System in a Rural Area: The Case of La Pocatière, Québec, in International Journal of Urban and Regional Research, Vol. 31, No. 1, pp. 1461-67, Blackwell Publishing, Oxford, UK.

5. Florida R, 2005. Cities and the Creative Class, London, Routledge

6. Fusch G. and Shapira P.,2005. Rethinking, Regional Innovation and Change. Path Dependency or Regional Breakthrough?, New York, Springer Science +, Collections :Economics of Science, Technology and Innovation, 30, Business Media, Inc., Springer eBooks collection. Business and economics (SpringlerLink)

7. Jean B. et al., 2009. Comprendre le Québec rural, Rimouski, Chaire de recherche du Canada en développement rural (UQAR), Dispoinble en ligne $:$ http://semaphore.uqar.ca/id/eprint/446

8. Jean B.,2010. Negotiating 'Rural' and Constructing a Development Process: Rural Development Planning in Quebec, Chapter 5 in Douglas, David (ed.), Rural Planning and Development in Canada, Nelson Education Ltd., Toronto, pp.134-149, ISBN 13: 978- 0-17-650081-8.

9. Jean B., 2006. The Rural Communities Studies in Quebec: From the 'folk society' monographic approach to the recent revival of community as place-based rural development, Journal of Rural and Community Development 1, 56-68, online journal published par Rural Development Institute, ISSN: 1712-8277, http://www.jrcd.ca

10. MAMROT (Ministère des Affaires municipales, des Régions et de l'Occupation du territoire ) «Politique Nationale de la ruralité, Gouvernement du Québec», http://www.mamrot.gouv.qc.ca /developpement-regional-et-rural/ruralite/politiquenationale-de-la-ruralite/

11. OECD ,2010. Québec, Canada, Examens de l'OCDE des politiques rurales, OECD Publishing, Paris.

12. Solidarité Rurale du Québec, 2010. Avis. Occupation du territoire. Pour un Québec fort de ses communautés, SRQ, Disponible en ligne. 